\title{
A New Method for Establishing Operative Channels in Unilateral Biportal Endoscopic Surgery: Technical Notes and Preliminary Results
}

\section{Jun Dai}

The Second Hospital of Soochow University

\section{Xiao-feng Liu}

The Second Affiliated Hospital of Soochow University

\section{Qian-liang Wang}

The Second Affiliated Hospital of Soochow University

\section{Yu-jian Peng}

The Second Affiliated Hospital of Soochow University

\section{Qian-zhong-yi Zhang}

The Second Affiliated Hospital of Soochow University

Jun Yan ( $\nabla$ cxyanjun@hotmail.com )

The Second Affiliated Hospital of Soochow University https://orcid.org/0000-0003-3640-1714

\section{Research Article}

Keywords: Minimally invasive surgery, Unilateral biportal endoscopy, Operative channel establishment, Degenerative disc disease

Posted Date: November 9th, 2021

DOI: https://doi.org/10.21203/rs.3.rs-1040213/v1

License: (c) (1) This work is licensed under a Creative Commons Attribution 4.0 International License. Read Full License 


\section{Abstract \\ Background}

At present, there are no new method for establishing operative channels for the unilateral biportal endoscopic(UBE)system.

\section{Objective}

This paper describes a new method for establishing operative channels in UBE surgery. Meanwhile, we assessed the preliminary efficacy of unilateral biportal endoscopic discectomy (UBED) in treating degenerative disc disease.

\section{Methods}

We retrospectively reviewed 52 patients who underwent UBED from February 2020 to August 2020 via our new method. The Oswestry Disability Index (ODI) and visual analogue scale (VAS) score were measured preoperatively and 1 month, 3 months, 6 months and 12 months postoperatively. Repeated measures one-way analysis of variance (RM-ANOVA) was performed to compare the pre-and postoperative VAS scores and ODIs. Post hoc pairwise comparisons (paired t-tests) was used to compare the postoperative VAS scores and ODIs.

\section{Results}

The postoperative ODIs and VAS scores of low back pain and leg pain were significantly lower than those before surgery, and differences were statistically significant (all $p<0.05$ ). Further pairwise comparisons brought out significant differences in the ODI at each time point after surgery (all $p<0.008$ ). Significant differences were found in the VAS score of leg pain between 1 month and 6 months, 1 month and 12 months, and 3 months and 12 months (all $p<0.008$ ). Pairwise comparisons in the VAS score of back pain between 1 month and 12 months differences $(p<0.008)$.

\section{Conclusion}

The modified channel establishment can provide a clear field of view in UBE surgery. UBED can yield a satisfactory curative effect in treating degenerative disc disease.

\section{Introduction}

The Global Burden of Disease identified low back pain (LBP) as the most common cause of disability worldwide, especially in ageing populations and in those regions where life expectancy is highest, with a significant annual cost in terms of morbidity, lost productivity, and quality-adjusted life years $(1,2)$. Despite the numerous and complicated factors related to LBP, estimates have suggested that approximately $40 \%$ of LBP cases are 
attributed to degenerative intervertebral disc disease(3).Among degenerative intervertebral disc disease, lumbar spinal stenosis and lumbar disc herniation are the most common.

Currently, treatment for degenerative disc disease includes conservative and surgical strategies. In general, for those in whom conservative measures fail, surgical options have proved to be effective for improving physical function $(4,5)$. Nevertheless, conventional open spinal fusion surgery increases the risk of degeneration in adjacent motion segments, as well as adjacent segment disease, and simultaneously causes damage to spinal structures, such as paraspinal muscles, bone, and ligaments(6-8). To avoid these negative consequences of open surgery, minimally invasive surgery is becoming widely acceptable for treating spinal disorders. UBE surgery allows direct central and foraminal neural decompression to be achieved, similar to conventional open decompression discectomy, through sufficiently safe procedures involving a magnified clear view and free handling of instruments(9-11). However, certain limitations, such as a steep learning curve, orientation under endoscopic vision, and capability to sufficiently decompress the spinal canal, limit the use of fully endoscopic techniques. (12) The surgical anatomy in biportal endoscopic spinal surgery is similar to that in conventional lumbar microdiscectomy, which is familiar to spinal surgeons converting from microsurgery to biportal surgery. The magnification of pathologic lesions via a 4-mm biportal endoscope and the provision of a clean operative field through continuous saline irrigation allow visualization of anatomical details during surgery. Nearly all microsurgical instruments, such as high-speed drills, pituitary forceps, and Kerrison punches, can also be utilized in biportal endoscopic surgery. Therefore, biportal endoscopic surgery is considered a minimally invasive technique with a shorter learning curve than any other minimally invasive spinal surgery technique, including fully endoscopic surgery.

At present, there are no special working sleeves available for the biportal endoscopic system. For convenient movement of the scope and instrument minimally invasive surgery needs to be performed in a cavity filled with irrigated saline between the lamina, flavum and paraspinal muscles. The clarity of visualization under the endoscope is affected by the size of the cavity and the water flow rate(9).

Here, we attempted to establish a modified working channel to easily expand and clarify the operational field in UBE surgery. The purpose of this study was to introduce UBE decompression surgery for degenerative disc disease using the modified working channel technique and present preliminary clinical results.

\section{Materials And Methods}

\section{Ethical consideration}

This retrospective study was approved by the research ethics committee of our hospital (JD-HG-2021-07). The study was conducted in accordance with the Declaration of Helsinki.

\section{Patient selection}

A total of 52 patients underwent UBED from February 2020 to August 2020. One patient was excluded due to a history of spinal surgery, and one was lost to follow-up. Finally, a total of 50 patients were included in this study. For an accurate diagnosis, the X-ray, CT, and MRI were performed in all patients. Every patient underwent singlesegment UBED. All patients were treated by a single experienced orthopaedic surgeon. The inclusion criteria were as follows: (1) single-level lumbar disc herniation or lumbar spinal stenosis; (2) preoperative symptoms of lower 
extremity radiating pain or LBP (VAS $\geq 4$ ) or neurogenic claudication; (3) failure of over 3 months of appropriate conservative treatment. The exclusion criteria were as follows: (1) spondylolytic spondylolisthesis or motion instability ( $>5^{\circ}$ of angulation or $>3 \mathrm{~mm}$ of translation); (2) infection, fracture, or previous spinal surgery; or (3) psychological condition (e.g., dementia, intellectual disability, or drug abuse).

\section{Modified channel establishment}

We took lumbar disc herniation(L4-5,left-sided interlaminar approach)as an example.A patient was placed in the prone position over the radiolucent chest frame in a flexed position. The surgeon standed in the left side of the patient. Two standard entry points were going to be marked at $1 \mathrm{~cm}$ above and below the disc space on an anteroposterior view. The cranial mark was assigned to viewing portal while caudal mark was assigned to working portal. The surgeon inserted the needles at two incision marks. Then, C-arm fluoroscopy was performed to ensure that the tips of the needles were in the ideal position (Fig. 1A). After withdrawing the needles, the skin and lumbodorsal fascia were incised in turn according to the incision marks. Two periosteal strippers were inserted into the incision vertically until they reached the surface of the lamina. To preliminary prepare the surgical field, the two periosteal strippers were used to strip soft tissue from the laminar surface until they met. Two vascular forceps were then used: one was inserted from the cranial incision (viewing portal), and the other was inserted from the caudal incision (working portal). The infusion strap was inserted through the cranial incision, passed below the muscle layer and exited from the caudal incision (Fig. 1B). A Y-shaped drainage device, which was made of suction apparatus tube and infusion tube, was fixed to the patient's skin with sutures to form a stable water flow channel at the caudal incision (Fig. 1C). In the case of water flow stoppage or bleeding in the surgical field, the assistant tightened the infusion strap to expand the space between lamina and paraspinal muscles(Fig. 1D). Raw materials and physical graph of the modified channel were illustrated and described in Fig. 2. Two examples of modified channel establishment was shown in the Supplementary Video (see Video, Supplemental Digital Content 1 and Supplemental Digital Content 2). The other procedures for UBED were the same as described by Choi KC et al(13).

\section{Postoperative evaluation}

After operation and before discharging from hospital, all patients were re-examined with MRI and CT scan. The images after surgery showed sufficient decompression of spinal cord and excluded acute complications. Clinical data were obtained for evaluation, including demographic data, operative data, and clinical outcome data. The operative data included the modified channel establishment time, biportal endoscopic time, postoperative hospital stay and incidence of complications. The modified channel establishment time was estimated from the moment of skin incision to the time of Y-shaped drainage device fixation to the patient's skin. The biportal endoscopic time was calculated from the first entry of the endoscope to the last extraction. Clinical outcomes were assessed using VAS score for back pain and leg pain, as well as the ODI. The ODI and VAS score were measured preoperatively and 1 month, 3 months, 6 months and 12 months postoperatively.

\section{Statistical analysis}

All statistical analyses were performed using SPSS version 20.0 (SPSS, Inc., Chicago, IL, USA). The Shapiro-Wilk test was used to evaluate the distribution of the collected data. Normally distributed continuous variables are presented as the mean and standard deviation (SD), whereas nonnormally distributed variables are presented as the median and interquartile range (IQR). Categorical variables are presented as numbers and percentages (\%). Repeated measures one-way analysis of variance (RM-ANOVA) was performed to compare the pre- and 
postoperative VAS scores and ODIs. Post hoc pairwise comparisons (paired t-tests) was used to compare the postoperative VAS scores and ODIs. A significance level of multiple comparisons ( $p$-value of $<0.008)$ was determined by Bonferroni correction.

\section{Results}

\section{Characteristics and demographic data}

A total of 50 patients were included in this study, consisting of 34 males (68.00\%) and 16 females (32.00\%). The average age of the patients included in this study was $34.48 \pm 8.89$ years old. The average body mass index (BMI) of the participants was $24.62 \pm 4.27 \mathrm{~kg} / \mathrm{m}^{2}$. The operative levels included $\mathrm{L} 4-5$ in 21 cases (42.00\%) and L5-S1 in 29 cases (58.00\%). 48 patients with lumbar disk herniation and 2 patients with lumbar spinal stenosis were enrolled in the study. The characteristics and demographic data of all included are presented in Table 1.

Table 1

Characteristics and demographic data

\begin{tabular}{|ll|}
\hline Characteristic & Value \\
\hline Age, mean \pm SD, years & $34.48 \pm 8.89$ \\
\hline Sex & $34(68.00 \%)$ \\
\hline Male & $16(32.00 \%)$ \\
\hline Female & $1.72 \pm 0.09$ \\
\hline Height, mean \pm SD, m & $73.10 \pm 17.03$ \\
\hline Weight, mean \pm SD, kg & $24.62 \pm 4.27$ \\
\hline BMI, mean \pm SD, kg/m² & \\
\hline Approach side & $28(56.00 \%)$ \\
\hline Left & $22(44.00 \%)$ \\
\hline Right & \\
\hline Operative segment & $21(42.00 \%)$ \\
\hline L4-L5 & $29(58.00 \%)$ \\
\hline L5-S1 & $48(96.00 \%)$ \\
\hline Disease & $2(4.00 \%)$ \\
\hline Lumbar disc herniation & \\
\hline Lumbar spinal stenosis & \\
\hline
\end{tabular}

BMI: body mass index

*All data are presented as $n$ (\%), unless otherwise specified

Operative data and complications 
The mean channel establishment time was $4.56 \pm 2.73$ minutes, and the mean biportal endoscopic time was $57.78 \pm 21.02$ minutes. There was 1 case of postoperative recurrence, 1 case of dural tear, and no cases of symptomatic epidural haematoma or infection. The patient who experienced recurrence of disc herniation one month after operation underwent additional revision surgery. The patient with a postoperative dural tear improved after conservative treatment. The operative data are summarized in Table 2.

Table 2

Operative data and complications

\begin{tabular}{|ll|}
\hline Parameter & Result \\
\hline $\begin{array}{l}\text { Channel establishment time, } \\
\text { mean } \pm \text { SD, min }\end{array}$ & $4.56 \pm 2.73$ \\
\hline $\begin{array}{l}\text { Biportal endoscopic time, } \\
\text { mean } \pm \text { SD, min }\end{array}$ & $57.78 \pm 21.02$ \\
\hline $\begin{array}{l}\text { Length of postoperative hospital stay, } \\
\text { mean } \pm \text { SD, days }\end{array}$ & $3.08 \pm 1.13$ \\
\hline Complications & \\
\hline Revision & $1(2.0 \%)$ \\
\hline Dura tear & $1(2.0 \%)$ \\
\hline Symptomatic epidural haematoma & 0 \\
\hline Infection & 0 \\
\hline
\end{tabular}

\section{Clinical outcomes}

The VAS scores for back pain improved from $5.20 \pm 2.57$ preoperatively to $1.96 \pm 0.95,1.50 \pm 0.84,1.64 \pm 1.08$ and $1.18 \pm 0.39$ at 1 month, 3 months, 6 months and 12 months postoperatively, respectively. The VAS scores for leg pain improved from $7.02 \pm 2.25$ preoperatively to $2.02 \pm 1.27,1.48 \pm 0.89,1.32 \pm 0.79$ and $0.88 \pm 0.52$. The ODI improved from $51.08 \pm 19.97$ preoperatively to $19.62 \pm 15.51,8.26 \pm 7.40$, and $7.54 \pm 6.42$ to $3.24 \pm$ 1.10 , respectively. The postoperative ODIs and VAS scores of low back pain and leg pain were significantly lower than those before surgery, and differences were statistically significant (all $p<0.05)$. Further pairwise comparisons brought out significant differences in the ODI at each time point after surgery (all $p<0.008$ ).

Significant differences were found in the VAS score of leg pain between 1 month and 6 months, 1 month and 12 months, and 3 months and 12 months (all $p<0.008$ ). Pairwise comparisons in the VAS score of back pain between 1 month and 12 months differences $(p<0.008)$. The clinical outcomes and complications are summarized in Table 3. 
Table 3

Clinical outcomes data

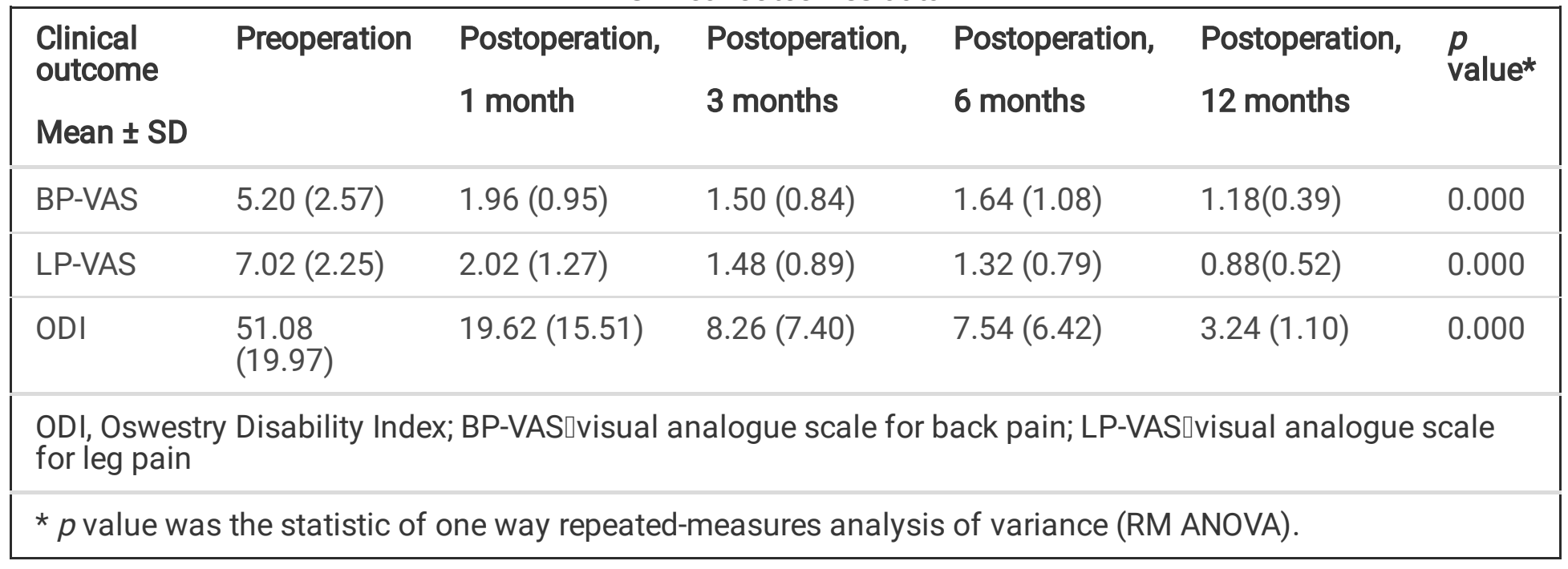

\section{Advantages of the modified channel method}

UBED requires water as a medium. Flowing water is beneficial for haemostasis and maintaining a clear field of view. Thus, continuous water flow is key to ensuring a smooth operation. If the water flow ceased, the surgical field of view became blurred (Fig. 3A; Fig. 3C). After tightening the infusion strap to expand the space between paraspinal muscles and lamina, the surgical field of vision again became clear (Fig. 3B; Fig. 3D).

\section{Discussion}

UBE surgery is a widely performed minimally invasive spinal surgery with satisfactory clinical outcomes, such as reduced pain and rapid recovery(14). Generally, specific instruments are required during conventional percutaneous endoscopic lumbar discectomy surgery, as well as shallow learning curve and a high rate of complications when applied for the treatment of lumbar spinal stenosis(15). The endoscopic instruments used in UBE could also be used in other surgeries, such as knee arthroscopy(8), greatly reducing medical expenses. Furthermore, the use of two channels, the viewing portal and the working portal, which are similar to those used in an interlaminar approach, is more in line with the surgical habits of spinal surgeons and allows full exposure of the area requiring decompression. However, there are still some shortcomings of UBE under certain conditions. On the one hand, it requires muscle splitting and shaving of the working space, which may cause bleeding affecting the surgical field and prolong the operative duration. On the other hand, laminotomy and foraminoplasty are usually performed in UBE by piezosurgery, and the high-frequency ultrasonication of piezosurgery causes the surgical field to become turbid, increasing the difficulty of the procedure for the surgeon.

Considering these disadvantages of UBE surgery, we designed a simple device for establishing the surgical portals. This device is versatile, allows a sufficient range of motion for surgical instruments and continuous irrigation, and is both readily available and relatively inexpensive. Such a device could be easily regulated by assistants, enabling prompt changes in the water flow volume and operating space. If the surgeon needs a clearer visual field under endoscopy or a temporary space for instrument movement, the assistant can gently tighten the strap to complete the operation (Fig. 3). Obviously, the convenience of this design is that the surgical 
space can be temporarily regulated without excessive muscle tissue ablation and the consequent minor damage. Meanwhile, the Y-shaped drainage device applied in our method provides a temporary fulcrum for the working portal, which is conducive to stable operation by the spinal surgeon and reduces the risks associated with equipment slippage. In addition, unlike stainless steel or titanium alloy, our drainage device is made from a soft plastic suction tube (an inexpensive material that is easy to trim), which can yield to the moving instruments while providing the stiff support required during surgery, thus increasing the convenience of the operation (Fig. 2). In addition, such a device could be temporarily fixed to the skin by silk thread without requiring long-term support from a surgical assistant.

Our study demonstrated that this modified portal design for UBE surgery was a feasible and effective technique for use in the treatment of single-level lumbar degenerative disease, including stenosis and disc herniation. The clinical outcomes of biportal endoscopic decompression at 12 months after surgery confirmed the advantages of UBE. As mentioned above, there were significant improvements in the mean VAS scores for leg pain and back pain, as well as in the ODIs during the follow-up period compared with preoperatively (Table 3 ). Furthermore, the endoscopic operative duration was 71.04 minutes per level, with an average channel establishment time of only 5.20 minutes (Table 2). In fact, the time for establishing a surgical portal notably decreased as experience was gained. This result also means that surgeons could quickly grasp the essentials of channel establishment with short-term training and avoid excessive increases in the operative duration, thereby reducing unnecessary damage to patients.

In addition, UBE surgery with the modified portal technique also showed less postoperative drainage (data not shown). The drainage tube was once routinely inserted into the operative cavity in the first few patients. However, we found that the amount of postoperative drainage was very small, and the extubation time was only one day after the operation. Therefore, whether the drainage tube needed to be placed was only dependent on the situation during our surgery. This result is not consistent with that of a previous study, which claimed that saline used for irrigation during the operation would infiltrate into the surrounding muscle and leak into the drain after surgery, which might be the reason for the greater drainage volume(16). This difference may be due to our shorter operative duration, the use of high-definition imaging, and the application of a radiofrequency probe and piezosurgery during the operation, all of which minimized the risk of bleeding in small vessels and cancellous bone. More importantly, the Y-shaped drainage device was conducive to the discharge of saline, thus greatly decreasing the remaining irrigation fluid. We also believe that reducing the need for drainage tube placement would support patient activity in the early postoperative period and the concept of rapid recovery from minimally invasive surgery.

Despite the potential superiority of UBE surgery, signs of muscle oedema were still detected on postoperative magnetic resonance imaging. Nevertheless, the complications were limited to one case of dural tear (2.0\%), one case of revision surgery (2.0\%), and there were no cases of symptomatic haematoma, infection (Table 2).

Several limitations of the current study should be addressed. First, all surgeries was done by a single surgeon. The relatively small sample size is another limitation. Moreover, to facilitate a standardized operation, hydromechanical tests should be performed to confirm the specific changes in the surgical area during use of the modified operative channel. Furthermore, the effect of the irrigation fluid volume on muscle or nerve roots, especially via shear stress, needs to be further studied in detail. 


\section{Conclusion}

This study is the first to describe a modified method for establishing operating channels in UBE surgery for single-level lumbar degenerative disease. This novel design enables better decompression and assistive manoeuvres that provide mechanical support and reduce bleeding, which are the most useful features. Therefore, for patients who are candidates for UBE surgery, our convenient method for establishing surgical portals is a feasible option.

\section{Abbreviations}

UBE: Unilateral biportal endoscopic system; UBED: Unilateral biportal endoscopic discectomy; ODI: Oswestry Disability Index; VAS: Visual analogue scale; RM-ANOVA: Repeated measures one-way analysis of variance; LBP: Low back pain

\section{Declarations}

\section{Acknowledgements}

The authors thank all of the participants in the Second Affiliated Hospital of Soochow University, and we thank the support of patients.

\section{Authors' contributions}

Jun Dai for writing- original draft preparationखXiao-feng Liu for methodology凶Qian-liang Wang for writing-

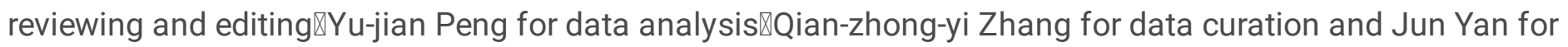
conceptualization. All authors read and approved the final manuscript.

\section{Funding}

This study is sponsored by the National Natural Science Foundation of China $(81971036,82002345,81902239)$, the Natural Science Foundation of Jiangsu Province (BK20191169), the Science and Technology Project of Suzhou (KJXW2019011) and the Preliminary Research Project of the Second Affiliated Hospital of Soochow University (SDFEYBS1905).

\section{Availability of data and material}

The data set supporting the conclusion of this article is available on request to the corresponding author.

\section{Ethics approval and consent to participate}

The retrospective study was approved by the Ethics Committee of the Second Affiliated Hospital of Soochow University $\rrbracket \mathrm{JD}-\mathrm{HG}$-2021-07】.Considering that this work was a retrospective study, the ethics committee waived the requirement for informed consent from patients.

\section{Consent for publication}

All authors agree on publication in this journal. 


\section{Competing interests}

All authors declare that they have no conflicts of interest and competing interest.

\section{Author details}

${ }^{1}$ Department of Orthopedic Surgery, The Second Affiliated Hospital of Soochow University, Suzhou, Jiangsu 215004, People's Republic of China.

\section{References}

1. Hoy D, March L, Brooks P, Blyth F, Woolf A, Bain C, et al. The global burden of low back pain: estimates from the Global Burden of Disease 2010 study. Ann Rheum Dis. 2014;73(6):968-74. https://doi.org/10.1136/annrheumdis-2013-204428.

2. Bydon M, Goyal A, Yolcu YU. Novel Intervertebral Technologies. Neurosurg Clin N Am. 2020;31(1):49-56. https://doi.org/10.1016/j.nec.2019.08.006.

3. Binch ALA, Fitzgerald JC, Growney EA, Barry F. Cell-based strategies for IVD repair: clinical progress and translational obstacles. Nat Rev Rheumatol. 2021;17(3):158-75. https://doi.org/10.1038/s41584-02000568-w.

4. Chen BL, Guo JB, Zhang HW, Zhang YJ, Zhu Y, Zhang J, et al. Surgical versus non-operative treatment for lumbar disc herniation: a systematic review and meta-analysis. Clin Rehabil. 2018;32(2):146-60. https://doi.org/10.1177/0269215517719952.

5. Ajiboye LO, Alimi M, Gbadegesin SA, Oboirien M. Treatment outcome of quality of life and clinical symptoms in patients with symptomatic lumbar degenerative disc diseases: which treatment modality is superior? Int Orthop. 2019;43(4):875-81. https://doi.org/10.1007/s00264-018-4248-5.

6. Telfeian AE. Transforaminal Endoscopic Surgery for Adjacent Segment Disease After Lumbar Fusion. World Neurosurg. 2017;97:231-5. https://doi.org/10.1016/j.wneu.2016.09.099.

7. Yamasaki K, Hoshino M, Omori K, Igarashi H, Nemoto Y, Tsuruta T, et al. Risk Factors of Adjacent Segment Disease After Transforaminal Inter-Body Fusion for Degenerative Lumbar Disease. Spine (Phila Pa 1976). 2017;42(2):E86-E92. https://doi.org/10.1097/BRS.0000000000001728.

8. Park SM, Park J, Jang HS, Heo YW, Han H, Kim HJ, et al. Biportal endoscopic versus microscopic lumbar decompressive laminectomy in patients with spinal stenosis: a randomized controlled trial. Spine J. 2020;20(2):156-65. https://doi.org/10.1016/j.spinee.2019.09.015.

9. Heo DH, Son SK, Eum JH, Park CK. Fully endoscopic lumbar interbody fusion using a percutaneous unilateral biportal endoscopic technique: technical note and preliminary clinical results. Neurosurg Focus. 2017;43(2):E8. https://doi.org/10.3171/2017.5.FOCUS17146.

10. Heo DH, Park CK. Clinical results of percutaneous biportal endoscopic lumbar interbody fusion with application of enhanced recovery after surgery. Neurosurg Focus. 2019;46(4):E18. https://doi.org/10.3171/2019.1.FOCUS18695.

11. Kim N, Jung SB. Percutaneous Unilateral Biportal Endoscopic Spine Surgery Using a 30-Degree Arthroscope in Patients With Severe Lumbar Spinal Stenosis: A Technical Note. Clin Spine Surg. 2019;32(8):324-9. https://doi.org/10.1097/BSD.0000000000000876. 
12. Heo DH, Quillo-Olvera J, Park CK. Can Percutaneous Biportal Endoscopic Surgery Achieve Enough Canal Decompression for Degenerative Lumbar Stenosis? Prospective Case-Control Study. World Neurosurg. 2018;120:e684-e9. https://doi.org/10.1016/j.wneu.2018.08.144.

13. Choi KC, Shim HK, Hwang JS, Shin SH, Lee DC, Jung HH, et al. Comparison of Surgical Invasiveness Between Microdiscectomy and 3 Different Endoscopic Discectomy Techniques for Lumbar Disc Herniation. World Neurosurg. 2018;116:e750-e8. https://doi.org/10.1016/j.wneu.2018.05.085.

14. Respino M, Jaywant A, Kuceyeski A, Victoria LW, Hoptman MJ, Scult MA, et al. The impact of white matter hyperintensities on the structural connectome in late-life depression: Relationship to executive functions. Neuroimage Clin. 2019;23:101852. https://doi.org/10.1016/j.nicl.2019.101852.

15. Sairyo K, Sakai T, Higashino K, Inoue M, Yasui N, Dezawa A. Complications of endoscopic lumbar decompression surgery. Minim Invasive Neurosurg. 2010;53(4):175-8. https://doi.org/10.1055/s-00301262814.

16. Wren SM, Schussler L, Burkle FM, Jr. Global Action Needed to Protect Humanitarian Surgeons and Patients During Wars and Armed Conflicts-Reply. JAMA Surg. 2020;155(1):90-1. https://doi.org/10.1001/jamasurg.2019.3501.

\section{Figures}



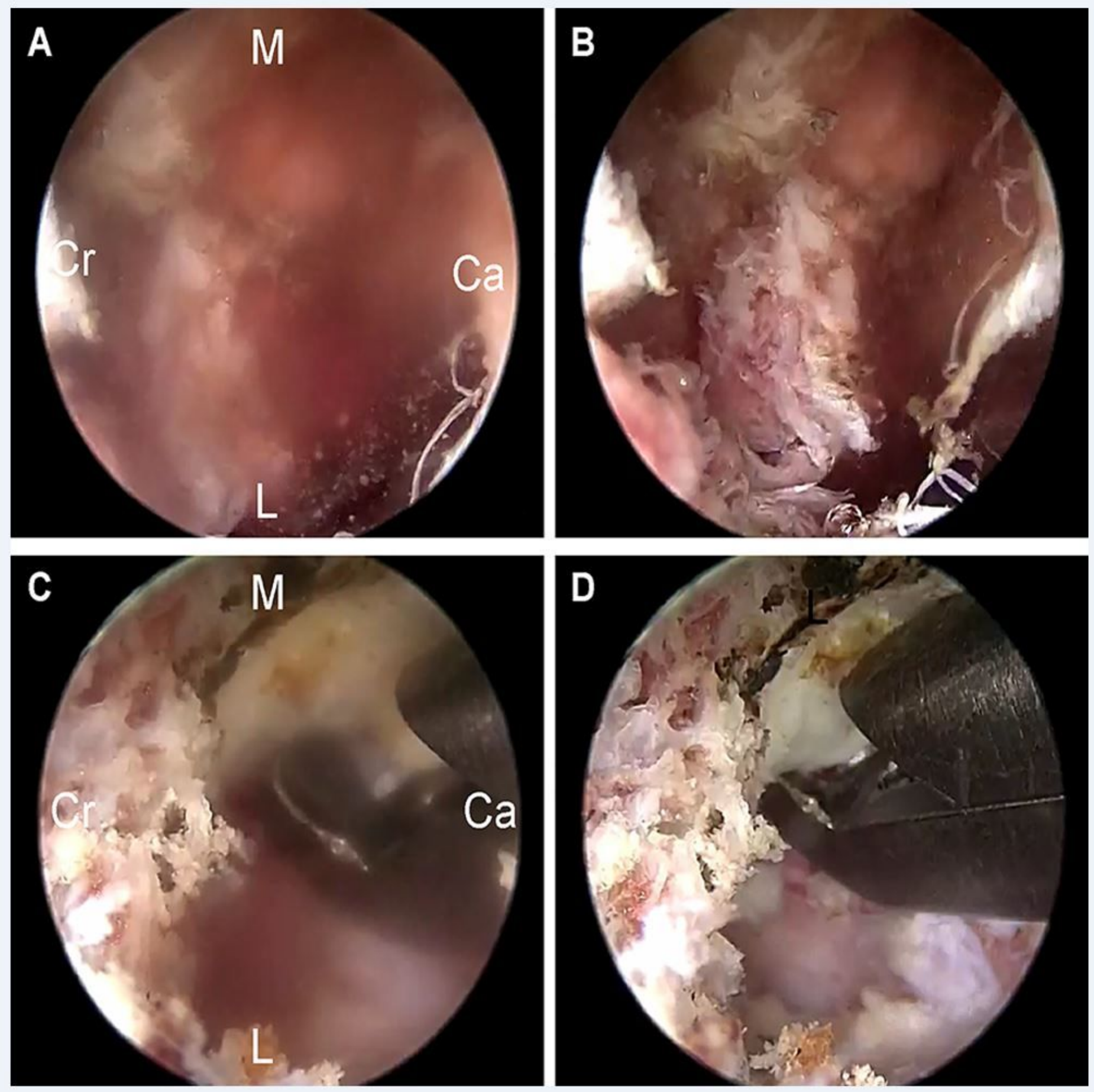

\section{Figure 1}

Procedures for modified channel establishment. (A) Localization of needles. (B) Insertion of the infusion strap through the cranial incision (viewing portal) and exit of the strap from the caudal incision (working portal) below the muscle layer. (C) Fixation of a Y-shaped drainage device to the patient's skin with sutures to form a stable water flow channel at the caudal incision. (D) Blurring of the surgical field of view when the water flow ceased, followed by clearance of the surgical field of view upon the assistant tightening the infusion strap to expand the interstitial space. 

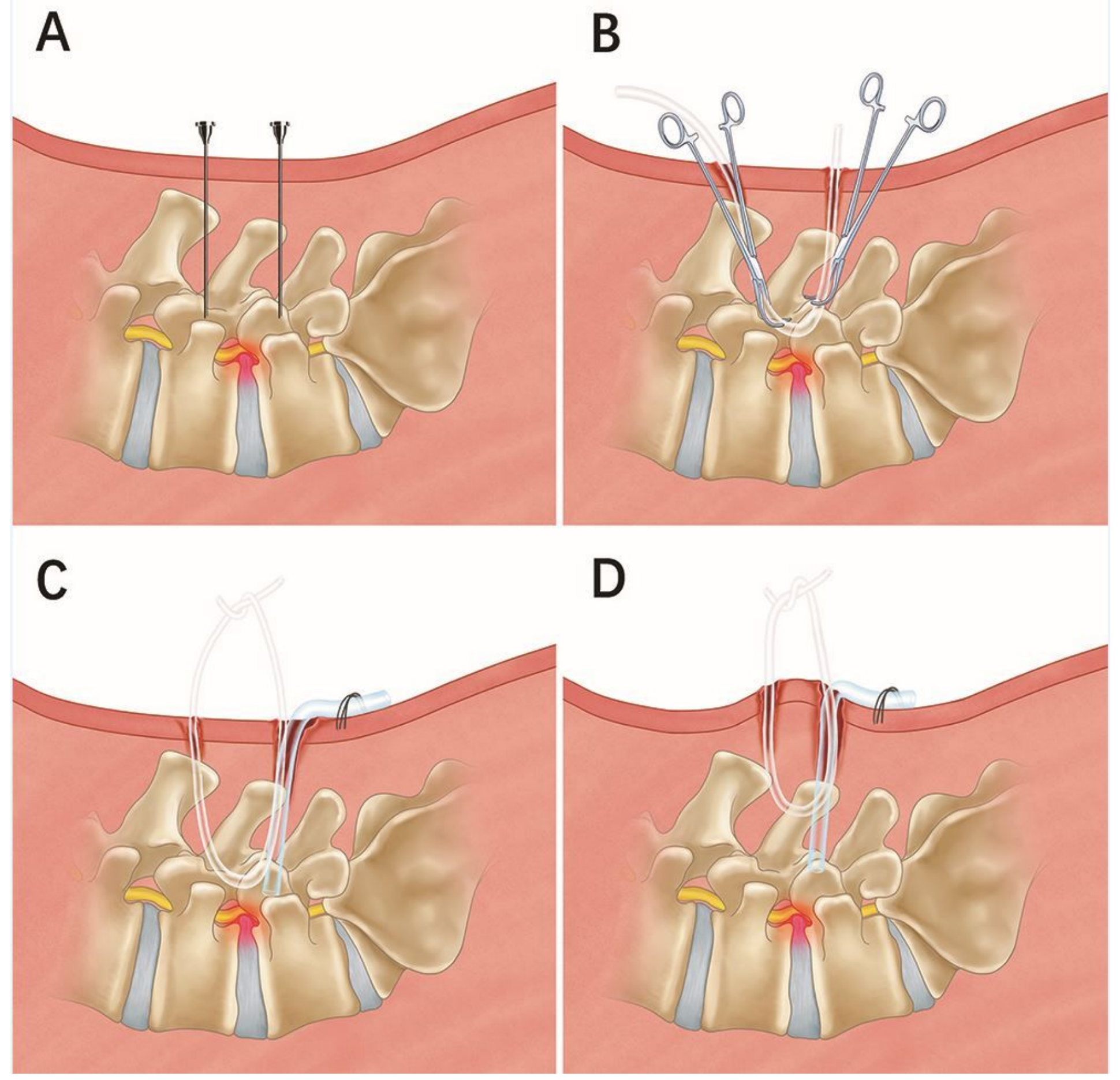

\section{Figure 2}

Raw materials and physical graph of the modified channel. (A) Infusion tube. (B) Infusion strap made by infusion tube. (C) Suction apparatus tube. (D) Y-shaped drainage device made of suction apparatus tube. (E) Physical graph of modified channel. $\Delta$ Fixation of a Y-shaped drainage device to the patient's skin with sutures. Onfusion strap was knotted above the skin. 

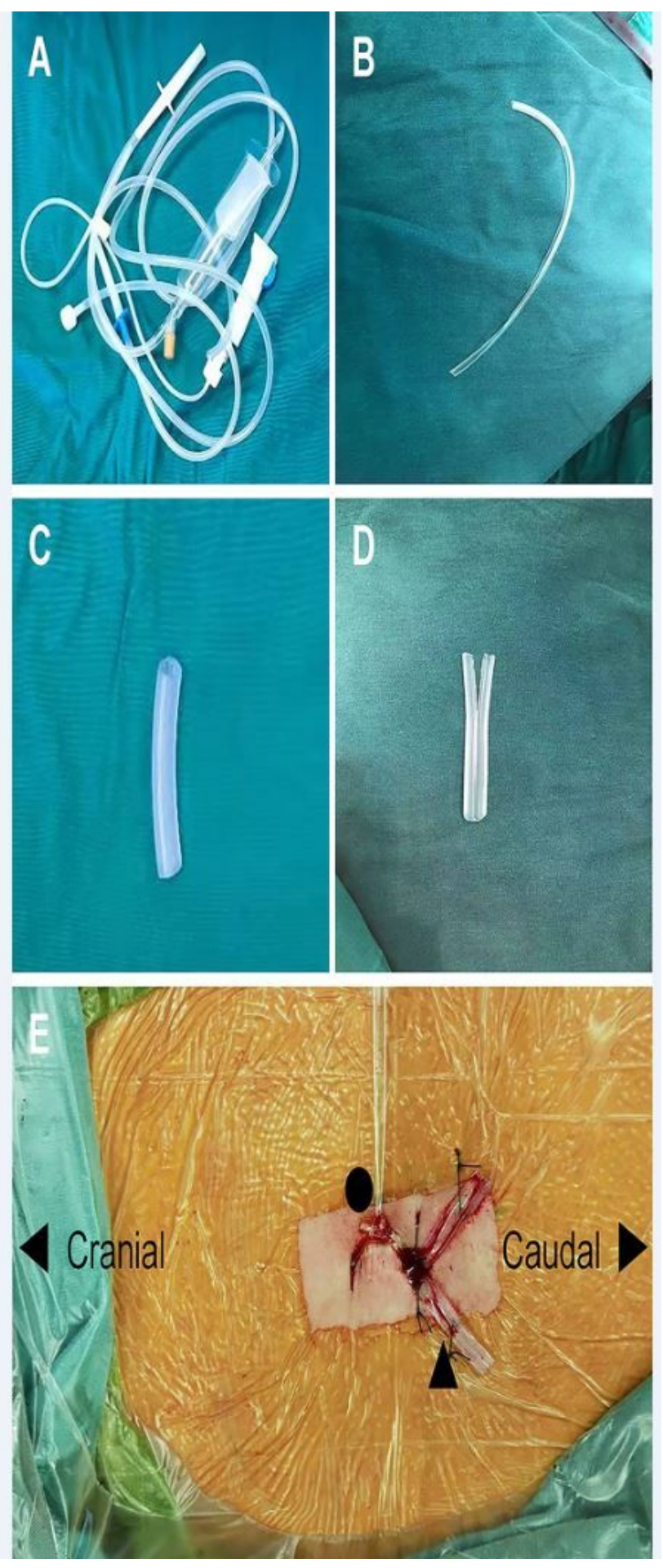

Figure 3

The endoscopic view changes. $(A, C)$ The endoscopic view was blurred due to the blockage of water flow. $(B, D)$ After tightening the infusion strap, the endoscopic view again became clear.Ca indicates caudal sides; $\mathrm{Cr}$, cranial sides; L,lateral; M, medial.

\section{Supplementary Files}

This is a list of supplementary files associated with this preprint. Click to download. 
- SupplementalDigitalContent1.mp4

- SupplementalDigitalContent2.mp4 